\title{
Wenn Kinder nicht zuhören: Auch an ADHS ...
}

W enn Kinder „nicht zuhören“ oder nur verzögert reagieren, wenn sie angesprochen werden, liegt der Verdacht auf eine Hörstörung nahe. Ergibt die pädaudiologische Untersuchung keine auffälligen Befunde, sollte der HNO-Arzt bei ausgeprägter motorischer Unruhe des Kindes sowie mangelnder Konzentration und Ausdauer auch an eine Aufmerksamkeitsstörung denken und eine entsprechende Diagnostik in die Wege leiten, empfahl Dr. Jochen Gehrmann von der Klinik für Kinder- und Jugendpsychiatrie am St. Marien- und St. Annastiftskrankenhaus Ludwigshafen.

Leitsymptome der ADHS (Aufmerksamkeitsdefizit-Hyperaktivitäts-Störung) sind Unaufmerksamkeit, Impulsivität und Hyperaktivität, die situationsübergreifend auftreten und mindestens sechs Monate anhalten: Eine ausdauernde, zielgerichtete Aufmerksamkeit fällt dem Kind schwer und es lässt sich leicht ablenken. Das Kind zeigt eine starke grobmotorische und feinmotorische Unruhe, bleibt kaum ruhig sitzen, ist ständig in Bewegung, nestelt und zupft an sich, seiner Kleidung oder anderen Gegenständen. Die Impulsivität äußert sich in riskantem Verhalten, das Kind stürzt häufig oder verletzt sich, kann Regeln nur schwer einhalten, hat wenig Geduld, eine geringe Frustrationstoleranz und ist häufigen Stimmungsschwankungen ausgesetzt.

Solche Auffälligkeiten machen sich oft schon im Vorschulalter bemerkbar. Ein starker Bewegungsdrang sei bei Kindern jedoch normal, und die Konzentrationsfähigkeit entwickle sich erst nach und nach, betonte Gehrmann. Eine spezifische ADHS-Diagnostik sollte daher ab dem Grundschulalter von erfahrenen Kinderund Jugendärzten oder -psychiatern durchgeführt werden.

\section{... und an die Auditive Verarbeitungs- und Wahr- nehmungsstörung denken!}

D ifferentialdiagnostisch von der ADHS abzugrenzen ist die Auditive Verarbeitungs- und Wahrnehmungsstörung (AVWS).

Bei der AVWS handelt es sich um eine Störung zentraler Prozesse des Hörens, erklärte Prof. Dr. Annerose Keilmann vom Schwerpunkt Kommunikationsstörungen der HNO-Universitätsklinik Mainz. Dazu zählen beispielsweise die binaurale Interaktion zur Geräuschlokalisation, die auditive Selektion, die dichotische Verarbeitung und das auditive Gedächtnis. Kinder mit AVWS haben vor allem in geräuschvollen Situationen Schwierigkeiten, sich auf relevante auditive Informationen zu konzentrieren. Sie haben Probleme, auditive, insbesondere sprachliche Informationen zu verstehen, missverstehen verbale Aufforderungen oder reagieren verzögert.

Bei Verdacht auf eine AVWS muss zunächst eine periphere Hörstörung ausgeschlossen werden, sagte Keilmann. Zusätzlich sei eine Binokularmikroskopie des Gehörgangs und des Trommelfells vorzunehmen.

Können zudem keine anderen Ursachen wie beispielsweise eine Aufmerksamkeitsstörung oder eine Intelligenzminderung die Symptome erklären, sollte eine AVWS-spezifische Diagnostik in die Wege geleitet werden. Diese sollte in einem spezialisierten Zentrum gemäß den Leitlinien der Deutschen Gesellschaft für Phoniatrie und Pädaudiologie (www.awmf.org/leitlinien/detail/ 1l/049-012.html) erfolgen. Die Testbatterie wird auf die individuellen Probleme des Kindes abgestimmt und prüft neben nonverbalen auch verbale Leistungen. Für die Diagnosestellung einer AVWS müssen die Leistungen in mindestens zwei Tests extrem unterdurchschnittlich sein, das heißt in dem Bereich liegen, der nur zwei Prozent aller Kinder umfasst.

Die Therapie richtet sich dann individuell nach den im Rahmen der Diagnostik festgestellten Defizite. Durch eine
Die Diagnostik und Therapie müsse auch Komorbiditäten berücksichtigen, die bei zwei Dritteln der Kinder mit ADHS auftreten. So leiden Kinder mit ADHS häufig unter Tics, Angststörungen, Schlafstörungen oder Störungen des Sozialverhaltens, berichtete Gehrmann.

Je nach Befund sei differenziert zu entscheiden, ob und welche Förderung und Behandlung das Kind benötigt. Das Therapiekonzept müsse multimodal ausgerichtet sein, sagte Gehrmann. Psychoedukation, Verhaltenstherapie, Elterntraining, eine Behandlung komorbider Störungen und bei schwererer Ausprägung eine individuell eingestellte Pharmakotherapie sind die wesentlichen Bausteine.

Gehrmann J. ADHS - was muss der HNO-Arzt wissen?

störungsspezifische Übungsbehandlung sollte versucht werden, Fähigkeiten wie Geräuschlokalisation, Lautidentifikation und auditive Merkfähigkeit zu verbessern, riet Keilmann. Zudem sollte das Kind lernen, wie es gestörte auditive Funktionen durch andere, insbesondere visuelle Sinneseindrücke kompensieren kann.

Hilfreich sind auch metakognitive und metalinguistische Strategien. Das Kind sollte beispielsweise dazu angehalten werden, sich auf Kernaussagen zu konzentrieren oder die Satzmelodie für die Unterscheidung von Fragen und Aufforderungen zu berücksichtigen.

Eine schalldämpfende Gestaltung des Klassenzimmers, kleine Lerngruppen oder zusätzliche schriftliche Materialien können dem Kind die Teilnahme am Schulunterricht erleichtern. Zudem erlauben die neuen Heilmittelrichtlinien die Verordnung einer FM-Anlage für Kinder mit gesicherter AVWS und reduziertem Sprachverstehen im Störgeräusch, berichtete Keilmann.

Keilmann A. Auditive Verarbeitungs- und Wahrnehmungsstörungen 